\title{
Multi-Robot Range-Only SLAM by Active Sensor Nodes for Urban Search and Rescue
}

Dali Sun, Alexander Kleiner and Thomas M. Wendt

\section{Post Print}

N.B.: When citing this work, cite the original article.

Original Publication:

Dali Sun, Alexander Kleiner and Thomas M. Wendt, Multi-Robot Range-Only SLAM by Active Sensor Nodes for Urban Search and Rescue, 2009, Robocup 2008: Robot Soccer World Cup XII, 318-330.

Postprint available at: Linköping University Electronic Press

http://urn.kb.se/resolve?urn=urn:nbn:se:liu:diva-72540 


\title{
Multi-Robot Range-Only SLAM by Active Sensor Nodes for Urban Search and Rescue
}

\author{
Dali Sun ${ }^{1}$, Alexander Kleiner ${ }^{1}$ and Thomas M. Wendt ${ }^{2}$ \\ ${ }^{1}$ Department of Computer Sciences \\ University of Freiburg \\ 79110 Freiburg, Germany \\ \{sun,kleiner\}@informatik.uni-freiburg.de \\ ${ }^{2}$ Department of Microsystems Engineering \\ University of Freiburg \\ 79110 Freiburg, Germany \\ wendt@imtek.uni-freiburg.de
}

\begin{abstract}
To jointly map an unknown environment with a team of autonomous robots is a challenging problem, particularly in large environments, as for example the devastated area after a disaster. Under such conditions standard methods for Simultaneous Localization And Mapping (SLAM) are difficult to apply due to possible misinterpretations of sensor data, leading to erroneous data association for loop closure. We consider the problem of multi-robot range-only SLAM for robot teams by solving the data association problem with wireless sensor nodes that we designed for this purpose. The memory of these nodes is utilized for the exchange of map data between multiple robots, facilitating loop-closures on jointly generated maps. We introduce RSLAM, which is a variant of FastSlam, extended for range-only measurements and the multi-robot case. Maps are generated from robot odometry and range estimates, which are computed from the RSSI (Received Signal Strength Indication). The proposed method has been extensively tested in USARSim, which serves as basis for the Virtual Robots competition at RoboCup, and by real-world experiments with a team of mobile robots. The presented results indicates that the approach is capable of building consistent maps in presence of real sensor noise, as well as to improve mapping results of multiple robots by data sharing.
\end{abstract}

\section{Introduction}

To jointly map an unknown environment with a team of autonomous robots is a challenging problem, particularly in large areas as for example the devastated area after a disaster. In USAR (Urban Search and Rescue) the mapping problem is generally harder, due to difficult operation conditions, such as unstructured environments and existing constraints for communication. However, the ability to map environments jointly is an essential requirement for coordinating robots and humans, such as first responders, within this domain. In urban environments GNSS (Global Navigation Satellite System) positioning is affected by the multipath propagation problem [6]. Buildings in the vicinity of the receiver reflect

\footnotetext{
${ }^{1}$ This research was partially supported by DFG as part of the collaborative research center SFB/TR-8 Spatial Cognition R7
} 
GNSS signals, resulting in secondary path propagations with longer propagation time, causing erroneous position estimates. Furthermore, long-range communication is perturbed by building structures made of reinforced concrete. Conventional methods for Simultaneous Localization And Mapping (SLAM), which require reliable data association for loop closure, are difficult to apply due to possible misinterpretations of sensor data. For example, bad visibility caused by smoke and fire affects vision-based tracking methods, and arbitrarily shaped structures from collapsed buildings require laser range finder-based data association to be fully carried out in 3D.

In this paper, we consider the problem of multi-robot SLAM for large robot teams in USAR. The data association problem is solved by using the unique IDs of deployed sensor nodes as features which are detectable in presence of low visibility via an omni-directional $2.4 \mathrm{GHz}$ antenna. Furthermore, the memory of these nodes is used for indirect communication, e.g. for the exchange of map data between multiple robots. More precise, robots subsequently store their position estimates of known sensor nodes into the memory of nodes within writing range. This information can be utilized by other robots for updating their individual map representations, facilitating loop-closures on jointly generated maps. In contrast to other approaches that require a priori a subset of sensor node locations that have to be surveyed in advance (so called anchor nodes), our method learns this locations stepwise from robots exploring the terrain. Moreover, new sensor nodes can arbitrarily be deployed during robot exploration, e.g. while searching for victims in an unknown environment.

The proposed method, named RSLAM, is a variant of FastSlam [16], extended for the multi-robot case and range-only measurements that are derived from the RSSI (Received Signal Strength Indication). We use a voting scheme for determining initial node locations from pairwise intersections of the signal strength measured at different robot locations [17]. The method is computational efficient, i.e. applicable in real-time, since it applies fast computable updates from robot odometry and rare range observations only. Each update is carried out in $O(n k)$, where $n$ is the number of sensor nodes and $k$ is the number of robot trajectories considered at the same time.

For our experiments we developed sensor nodes [20] meeting the ZigBee specification [8]. They are equipped with three sensors, measuring air pressure, temperature, and node orientation. The first two sensors might be utilized for user applications, such as monitoring the temperature and the air pressure during crisis management. Measurements of the node's orientation are useful for detecting the alignment of the antenna, which might be used for improving the RSSI in order to increase the accuracy of distance measurements.

The proposed method has been extensively tested in the USARSim [1] simulation environment which serves as basis for the Virtual Robots competition at RoboCup. We modified the simulator in that it provides range readings of virtual sensor nodes with respect to a model for signal path attenuation [18], which has been parameterized according to the developed hardware. The simulation results show that our method is capable to successfully map diverse types 
of environments by robot teams, also indoors, where signal strength has been heavily perturbed by walls. Finally, we present results from a real-world experiment demonstrating the capability of our system to handle communications of multiple nodes at the same time, as well as to deal with real sensor noise.

The remainder of this paper is structured as follows. In Section 2 related work is discussed, and in Section 3 the real system and the simulation system utilized for experiments are described. In Section 4 the sensor model, and in sections 5 the introduced SLAM method are discussed. Finally, we present experimental results in Section 6 and draw conclusions in Section 7.

\section{Related Work}

Inspired by the fundamental work of Smith et al. [19], early work on SLAM was mainly based on the Extended Kalman Filter (EKF) [3]. In connection with radio transmitters, the SLAM problem has been addressed as "range-only" SLAM [10, 4] since the bearing of the radio signal cannot accurately be determined. RFIDs have been successfully utilized for localizing mobile robots [7] and emergency responders [9,15]. Hähnel et al. [7] successfully utilized Markov localization for localizing a mobile robot in an office environment. Their approach deals with the problem of localization in a map previously learned from laser range data and known RFID positions, whereas the work presented in this paper describes a solution that performs RFID-based localization and mapping simultaneously during exploration. Also sensor networks-based Markov localization for emergency response has been studied [9]. In this work, existing sensor nodes in a building are utilized for both localization and computation of a temperature gradient from local sensor node measurements. Miller and colleagues examined the usability of various RFID systems for the localization of first responders in different building classes [15]. During their experiments, persons were tracked with a Dead Reckoning Module (DRM). In former work we introduced RFIDSLAM, an extension of graph-based SLAM for networks of RFIDs [11]. Furthermore, we demonstrated the efficient deployment of sensor nodes for multi-robot coordination and exploration [21].

\section{Test platform}

The test platform utilized for experiments is based on a team of 4WD differentially steered Zerg robots, as depicted in Figure 1(a). Each robot is equipped with a Sick S30-B Laser Range Finder (LRF), an Inertial Measurement Unit (IMU), over-constrained odometry for wheel-slippage detection, and a mobile sensor node.

The simulated counter part (see Figure 1(b)) has been designed for the USARSim simulator developed at the University of Pittsburgh [2]. USARSim, which serves as a basis for the RoboCup Rescue virtual competition, allows realistic simulations of raw sensor data and robot actuators, which can directly be accessed via a TCP/IP interface. Sensors, such as odometry and LRF, can be 


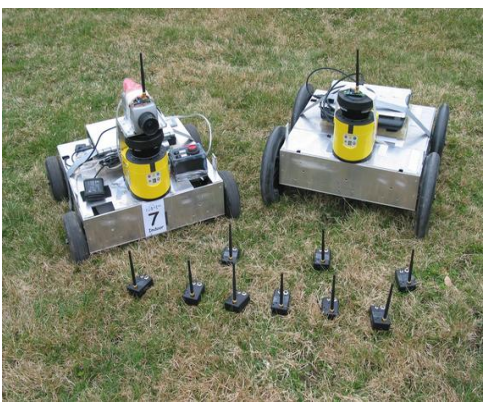

(a)

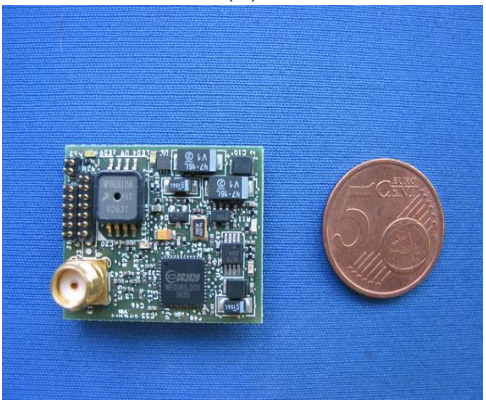

(c)

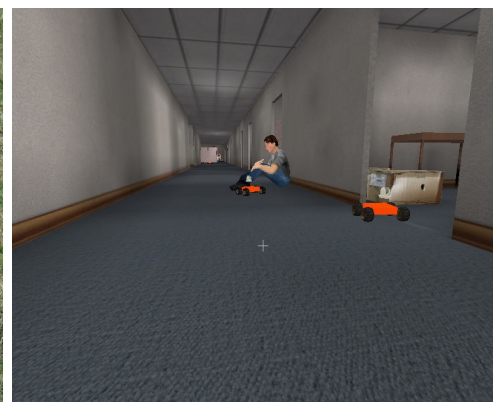

(b)

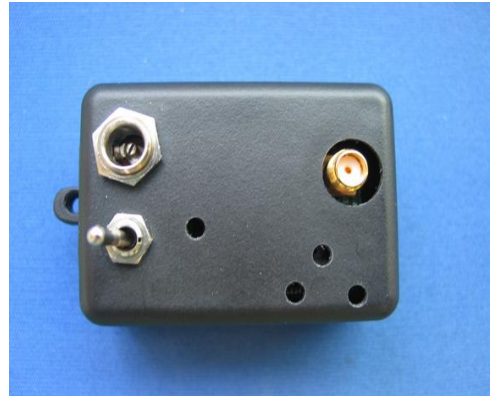

(d)

Fig. 1. (a) A team of Zerg robots equipped with antennas for sensor node communication, and (b) the corresponding simulation model in USARSim (b). (c-d) The designed sensor node: (a) the PCB, and (b) with housing.

simulated with the same parameters as they are found on real robots. We modified the simulator in that it provides range readings of virtual sensor nodes with respect to a model of signal path attenuation [18] (see Section 4). The model considers obstacles, such as walls and trees, that are located between two transceivers. Obstacles are detected by a ray tracing operation that is applied each time two transceivers are within communication range. Both platforms, the real and the simulated robot, achieved the first place during the RoboCup 2005 Rescue Autonomy competition, and the RoboCup 2006 Virtual Robots competition, respectively $[12,13]$.

We developed wireless sensor nodes based on the TI CC2420 transceiver [8] that meets the ZigBee specification. The transceiver implements an anti-collision protocol allowing the simultaneous reading of multiple nodes within range. The printed circuit board (PCB) is shown in Figure 1(c), and the sensor node with housing and omni-directional antenna is shown in Figure 1(a,d). An on board eight-bit micro controller [14] monitors the sensors on the PCB and establishes the communication link via the transceiver. The communication protocol is tailored for power minimization and transparent access to the radio link. Furthermore, the sensor node is equipped with three sensors, measuring air pressure, temperature, and antenna orientation, which might be utilized for user applica- 
tions. Measurements of the antenna orientation, for example, can be considered to improve the reliability of communication. RSSI and Link Quality Indication (LQI) are directly read from the transceiver chip and are directly transmitted to the reader.

In order to enable communication between several sensor nodes at the same time, a random broadcast scheme has been implemented. If the air channel is occupied heavily by radio traffic, nodes are sleeping for a random amount of time before re-sending their data. Alternatively, we experimented with a polling mechanism triggered by a master node that requests data packages within fixed cycles from each node, which led to a high latency time of the system. With the random broadcast scheme, we measured an average data rate of $28.6 \mathrm{~Hz}$ for one, $27.5 \mathrm{~Hz}$ for four, and $16.5 \mathrm{~Hz}$ for eight nodes transmitting at the same time. This data rate is sufficient also for mobile platforms since we assume a node density of maximally 4 nodes being in communication range at any location. Finally, we determined experimentally a maximal range of up to 35 meters for performing reliable communications.

\section{Sensor model}

The Transceiver-Receiver (TR) separation, i.e. the distance between an observed sensor node and the detector, can generally be estimated from the power of the signal known as RSSI (Received Signal Strength Indication). The sensor model describes the relation between the RSSI and range estimates denoted by distance $r$ an variance $\sigma_{r}$. Signal propagation, particularly in indoor environments, is perturbed by damping and reflections of the radio waves. Since these perturbations can depend on the layout of the building, the construction material used, and the number and type of nearby objects, modeling the relation between signal path attenuation and TR separation is a challenging problem.

Seidel and Rapport introduced a model for path attenuation prediction that can also be parameterized for different building types and the number of walls between transceiver and receiver [18]. The model relates the signal power $P$ to distance $d$ in the following way:

$$
P(d)[d B]=p\left(d_{0}\right)[d B]-10 n \log \frac{d}{d_{0}}+X_{\sigma}[d B],
$$

where $p\left(d_{0}\right)$ is the signal power at reference distance $d_{0}, n$ is the mean path loss exponent that depends on the structure of the environment, and $X_{\sigma}$ is the standard deviation of the signal.

During practical experiments we noticed that signal strength measurements of the used transceiver contain a high amount of outliers. Therefore, we applied RANSAC [5], which is an iterative method to estimate model parameters from a set of observations containing outliers. The parameter we are interested in is the average signal strength given a set of measurements. Therefore, a subset of measurements is randomly sampled, the average computed, and finally all the other measurements are tested against this average. If measurements fit well to 


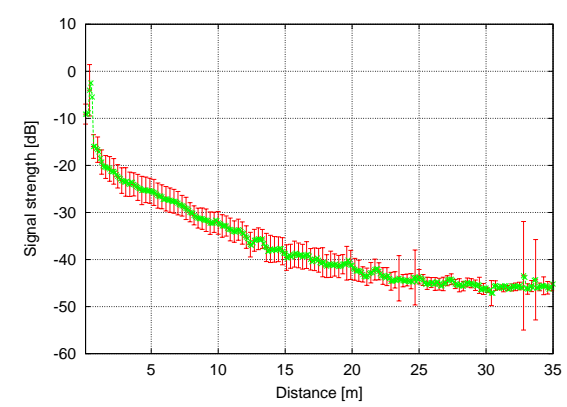

(a)

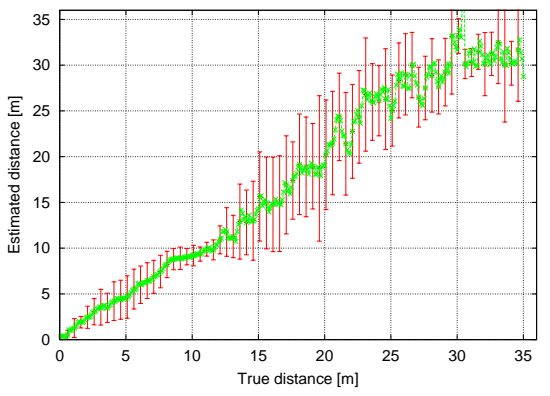

(b)

Fig. 2. (a) Measured signal strength at varying distance. (b) Relation between estimated distance and real distance. Each data point has been averaged from ten measurements, green points denote the computed mean and red bars the standard deviation.

the average, they are added to the subset, and rejected otherwise. The procedure is repeated iteratively until the subset contains sufficient data points.

We conducted several measurements for determining the model parameters of the described $2.4 \mathrm{GHz}$ sensor nodes in the testing environment, which are $p\left(d_{0}\right)=-12 d B$, and $n=2$. The standard deviation $X_{\sigma}$ is taken from a lookup table that has been determined experimentally, as shown in Figure 2(a). Figure 2(a) depicts the mean and variance of the measured signal strength at different distances averaged over 10 measurements at each location. Figure 2(b) compares the estimate of the parameterized model from Equation 1 with ground truth.

\section{RFID SLAM}

We utilize FastSlam [16] in order to compute simultaneously the locations of the robot and of the sensor nodes. FastSlam estimates simultaneously the robot path $l_{1}, l_{2}, \ldots, l_{t}$ and beacon locations $b_{1}, b_{2}, \ldots, b_{N}$, where $l_{i}=\left(x_{i}, y_{i}, \theta_{i}\right)$, and $b_{j}=\left(x_{j}, y_{j}\right)$. The path of the robot is estimated by using a particle filter with $M$ particles, whereas each particle possesses its own set of Extended Kalman Filters (EKFs). EKFs are estimating beacon locations independently from each other conditioned on the robot path. Hence, there are $M \times N$ low-dimensional EKFs updated by the system.

We denote each EKF's state vector by $s_{i}=\left(b_{x}, b_{y}\right)^{T}$, and the corresponding $2 \times 2$ covariance matrix by $\Sigma_{s_{i}}$, where $\left(b_{x}, b_{y}\right)^{T}$ is the location of beacon $i$. From an observation $z=r$ of a landmark at range $r$ with covariance $\sigma_{z}$ (see Section 4), each particle is updated as follows: first, the observation is associated to one of the EKFs stored in the particle based on the unique ID of the detected nodes. 
Second, the observation is predicted by the following measurement function:

$$
h_{i}(s)=\left(\sqrt{\left(r_{x}-b_{x}\right)^{2}+\left(r_{y}-b_{y}\right)^{2}}\right) .
$$

Third, the associated state vector is updated from the observation by computing the innovation $v$ and covariance $\sigma_{v}$ by applying the law of error propagation:

$$
\begin{aligned}
v_{i} & =z-h_{i}(s) \\
\sigma_{v_{i}} & =\nabla h_{s} \Sigma_{s} \nabla h_{s}^{T}+\sigma_{z},
\end{aligned}
$$

where $\nabla h_{s}$ is the Jacobian matrix:

$$
\nabla h_{s}=\left(-\frac{\Delta x}{d},-\frac{\Delta y}{d}\right),
$$

where $\Delta x=r_{x}-b_{x}, \Delta y=r_{y}-b_{y}$, and $d=\sqrt{\Delta x^{2}+\Delta y^{2}}$. Then, the following Kalman update is applied:

$$
\begin{array}{r}
K=\Sigma_{s_{i}} \nabla h_{s}^{T} \sigma_{v_{i}}^{-1} \\
s_{i+1}=s_{i}+K v_{i} \\
\Sigma_{s_{i+1}}=\Sigma_{s_{i}}-K \sigma_{v_{i}} K^{T} .
\end{array}
$$

Finally, we update from each observation an importance weight for each particle based on the Mahalanobis distance between the measurement and the prediction:

$$
w_{i+1}=w_{i}-v_{i}^{T} \sigma_{v_{i}}^{-1} v_{i} .
$$

During each cycle, these weights are utilized to stochastically sample robot paths that best explain the measured ranges. According to the particle filtering framework, these particles are furthermore propagated based on the motion model of the robot if steering commands occur [16].

\subsection{EKF initialization}

One difficulty in range-only SLAM is to estimate the initial beacon locations. This is particularly important since beacon observations do not contain bearing information as required by SLAM methods in general. We use a voting scheme for determining initial node locations from pairwise intersections of range measurements at different robot locations [17]. This is carried out by maintaining a grid for each beacon, where each cell represents the probability that the beacon is located at the corresponding location. The grid is updated by a likelihood function $f\left(o_{i}, z_{i}\right)$ that assigns to each cell a probability with respect to the robot's pose estimate from odometry $o_{i}$ and range observation $z_{i}$. Given range measurements, the likelihood function generates circular probability distributions centered at the current pose of the robot, and width according to the confidence of the observation. After integrating several observations, the most likely beacon location can be determined by taking the maximum over all grid cells. The location at this maximum is taken to initialize one EKF for each particle. 


\subsection{Multi-robot mapping}

The memory of the sensor nodes is used for indirect communication, e.g. for the exchange of map data between multiple robots. If sensor node $k$ is within writing distance of robot $R$, its current map estimate, consisting of $n_{R}$ known beacon locations, is stored into the local memory $M_{k}$ of the node:

$$
M_{k} \leftarrow M_{k} \cup\left\langle j, b_{j}, \Sigma_{b_{j}}, R\right\rangle, \quad \text { for all } j \in n_{R}
$$

The stored data is utilized by other robots for updating their individual maps. This is carried out by calculating the local offset between individual robot maps from the relative pose displacement to common sensor nodes. Note that we assume that the IMU angle of each robot is aligned to magnetic north leading to displacements without angular components.

Map data from other robots can significantly accelerate the convergence of the map from a single robot. First, the initialization procedure described in Section 5.1 is not required for nodes that are observed the first time, if their location estimate is already known. Second, known sensor node locations can directly be updated by the EKFs, facilitating loop closure on jointly generated maps. Note that the update procedure automatically degrades from SLAM to ocalization if many of the communicated location estimates have tight covariance bounds, i.e. are close to ground truth.

\section{Results}

RSLAM has been tested in various USARSim environments generated by the National Institute of Standards and Technology (NIST). They provide both indoor and outdoor scenarios of the size bigger than $1000 \mathrm{~m}^{2}$, reconstructing the situation after a disaster. Furthermore, we conducted real-world experiments showing the capability of the developed sensor nodes to handle communications of multiple clients at the same time, as well as the capability of the proposed approach to deal with real sensor noise.

Figure 3 compares visually the mapping result from wheel-odometry and RSLAM, which has been carried out by aligning measurements from the LRF according to the pose estimates. The map generated from odometry is unusable for navigation, whereas the map generated by the introduced method is close to ground truth. For this experiment the map $\mathrm{RCO}^{7}$-Mapping has been used, which is also listed in Table 1.

Table 1 summarizes the results from applying the method on simulated indoor and outdoor maps. Maps starting with $\mathrm{RCO6}$, and $\mathrm{RCO}^{7}$ were introduced by NIST for RoboCup 2006 and 2007, respectively. Ground truth, i.e. the true trajectories of the robots, is directly provided by the simulator. The results show that single RSLAM successfully improves maps generated from odometry. Moreover, multi RSLAM, i.e. taking advantage of map data stored by other robots into node memories, yields even better results than single RSLAM. 


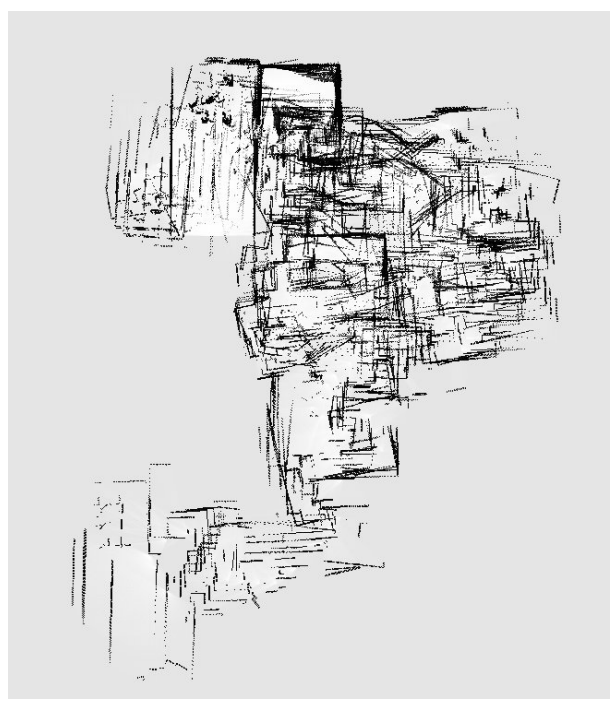

(a)

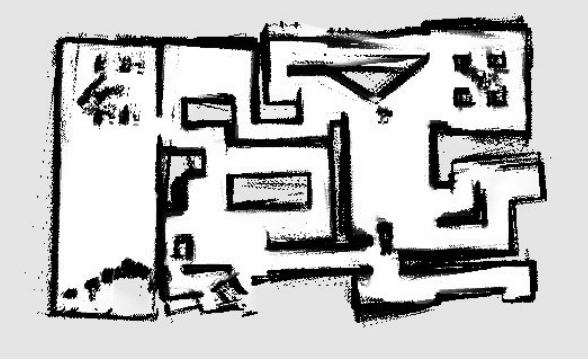

(b)

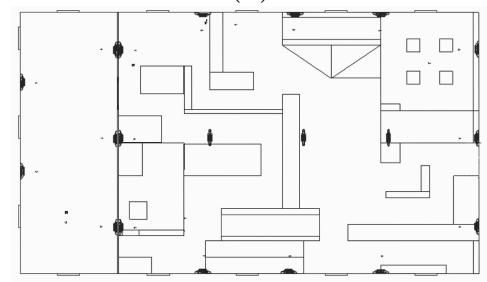

(c)

Fig. 3. Indoor map generated from wheel odometry (a), and multi RSLAM (b). The ground truth image has been provided by NIST (c).

Table 2 summarizes the results from real-robot experiments. During this experiment real sensor nodes have been distributed at fixed height and with fixed orientation in an outdoor environment. Four robots were manually steered ${ }^{1}$ through the environment while computing map estimates, i.e. the robot path and sensor node location, online. This information was used by subsequent robots for initializing their particle filters. Also during this experiment, maps generated from wheel odometry have been significantly improved by applying RSLAM. As can be seen by the third row, map data provided by other robots helps to increase this improvement. Due to limited accuracy of range readings computed from signal strength, a minimal possible positioning error around one meter has been reached. Figure 4 depicts the true positions and the positions finally estimated by the robots. During all experiments, an average computation time of $20 \mathrm{~ms}$ on a Pentium $41 \mathrm{GHz}$ has been measured.

\section{Conclusions and Future Works}

In this paper we presented RSLAM, an approach for multi-robot range-only SLAM based on sensor nodes for Urban Search and Rescue. We demonstrated recently developed sensor nodes and their capabilities in terms of signal strength detection and multi-sensor communication. Furthermore, we showed that RSLAM

\footnotetext{
${ }^{1}$ Note that two robots have been steered two times sequentially.
} 


\begin{tabular}{|l|c|c|c|c|c|}
\hline Map name (size in $\left[\mathrm{m}^{2}\right]$ ) & Robot & Odometry [m] & $\begin{array}{c}\text { RSLAM [m] } \\
\text { (single) }\end{array}$ & $\begin{array}{c}\text { RSLAM [m] } \\
\text { (multi) }\end{array}$ & \# RFID \\
\hline \hline \multirow{2}{*}{ RC07-Plywood (109) } & 1st & $4.7 \pm 3.4$ & $0.3 \pm 0.9$ & $0.3 \pm 0.9$ & \multirow{2}{*}{ 2nd } \\
& $3.5 \pm 2.6$ & $0.8 \pm 1.3$ & $0.6 \pm 1.1$ & \\
\hline \multirow{2}{*}{ RC07-Mapping (250) } & 1st & $7.0 \pm 4.9$ & $0.5 \pm 1.1$ & $0.5 \pm 1.1$ & \multirow{2}{*}{8} \\
& 2nd & $4.1 \pm 3.0$ & $0.6 \pm 1.4$ & $0.3 \pm 0.8$ & \\
\hline \multirow{2}{*}{ RC06-Day4a (846) } & 1st & $8.2 \pm 3.2$ & $0.4 \pm 0.6$ & $0.4 \pm 0.6$ & \multirow{2}{*}{14} \\
& 2nd & $13.2 \pm 5.8$ & $2.0 \pm 1.9$ & $0.8 \pm 1.5$ & \\
\hline \multirow{2}{*}{ RC07-Factory (1975) } & 1st & $17.4 \pm 8.3$ & $2.1 \pm 2.8$ & $2.1 \pm 2.8$ & \multirow{2}{*}{58} \\
& 2nd & $28.2 \pm 9.6$ & $4.6 \pm 3.6$ & $1.6 \pm 2.5$ & \\
\hline \multirow{2}{*}{ RC07-Mobility (3819) } & 1st & $12.9 \pm 6.9$ & $1.4 \pm 2.7$ & $1.4 \pm 2.7$ & \multirow{2}{*}{58} \\
& 2nd & $13.3 \pm 6.3$ & $1.7 \pm 3.0$ & $1.3 \pm 2.3$ & \\
\hline \multirow{2}{*}{ RC06-Day4b (4529) } & 1st & $5.0 \pm 3.3$ & $0.5 \pm 0.9$ & $0.5 \pm 0.9$ & \\
& 2nd & $6.7 \pm 4.3$ & $1.2 \pm 1.2$ & $0.3 \pm 1.0$ & \multirow{2}{*}{60} \\
& 3rd & $5.2 \pm 2.7$ & $0.4 \pm 0.8$ & $0.1 \pm 0.4$ & \\
\hline
\end{tabular}

Table 1. Avg. Cartesian positioning errors: Comparison between wheel odometry, single-robot RSLAM, and multi-robot RSLAM applied on different USARSim maps. Values are averaged from the total trajectory of each robot. Ground truth has been directly taken from the simulator.

\begin{tabular}{|c||c|c|c|c|c|c|}
\hline & Odo. $[\mathrm{m}]$ & $\begin{array}{c}\text { RSLAM [m] } \\
\text { (single) }\end{array}$ & $\begin{array}{c}\text { RSLAM [m] } \\
\text { (multi) }\end{array}$ & Speed [m/s] & Dist [m] \# RFID & \# \\
\hline \hline 1st robot & $8.6 \pm 6.0$ & $1.1 \pm 0.6$ & $1.1 \pm 0.6$ & 1.0 & 410 & \\
2nd robot & $5.0 \pm 4.0$ & $2.1 \pm 1.9$ & $1.3 \pm 0.9$ & 1.2 & 348 & 9 \\
3rd robot & $7.1 \pm 4.5$ & $2.2 \pm 1.7$ & $1.3 \pm 1.0$ & 1.3 & 320 & \\
4th robot & $5.3 \pm 3.2$ & $1.9 \pm 0.9$ & $1.5 \pm 0.6$ & 1.5 & 282 & \\
\hline
\end{tabular}

Table 2. Avg. Cartesian positioning errors: Comparison between wheel odometry and RSLAM applied on real robots. Ground truth has been manually generated by georeferencing.

allows it to a team of robots to map efficiently large areas under severe communication and operational constraints. Our results from simulation and real-word experiments show that robots can successfully correct noisy odometry readings and jointly improve their map estimates based on the wireless nodes. Moreover, we have shown that with multi RSLAM individual robots consistently gain better mapping results by data sharing via sensor nodes than carrying out the mapping task on their own.

Sensor nodes greatly simplify the task of multi-robot SLAM in three ways: First, features can be uniquely identified, solving trivially data association problems. Second, the number of features is low w.r.t. visual features and thus the SLAM problem is tractable even for large areas. Third, node memories can be used for indirect communication allowing robots to jointly correct their local maps. 


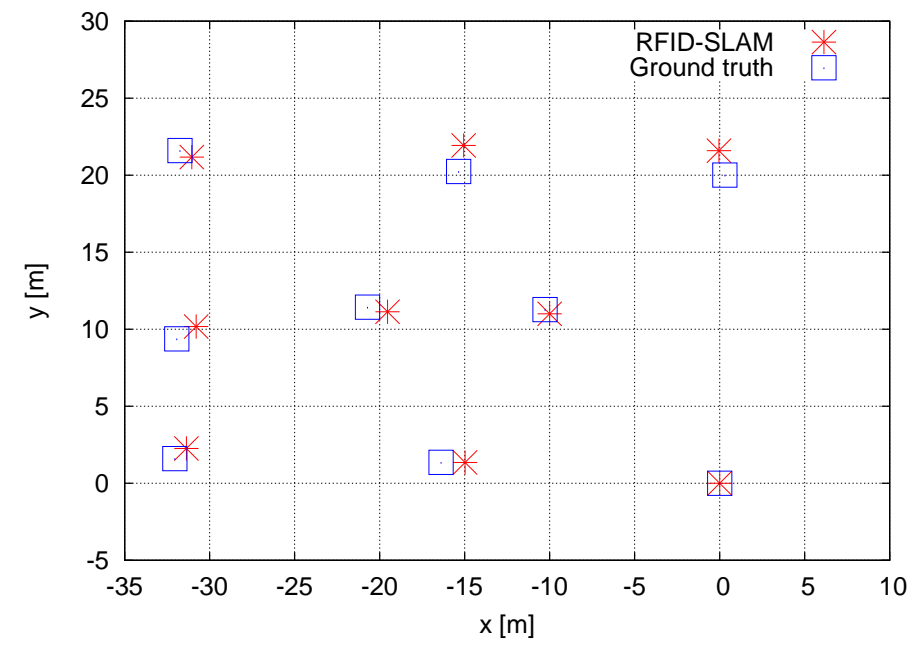

Fig. 4. Real-robot experiment: Comparison of the locations of the deployed real sensor nodes with manually measured ground truth

In future work we are planning to extend the approach by inter-node communication allowing nodes to improve their estimates in a decentralized manner, and to synchronize map data with other nodes that are in direct communication range. During our experiments we observed high variations of distance measurements when orientation and height of sensor nodes have been modified. Our future goal is to address these problems by integrating sensor measurements from the nodes, such as orientation and height. This will allow to generate specific sensor models for each node leading to more accurate distance estimations.

\section{References}

1. S. Balakirsky, C. Scrapper, S. Carpin, and M. Lewis. "USARSim: providing a framework for multi-robot performance evaluation". In Proceedings of PerMIS 2006, 2006.

2. S. Carpin, M. Lewis, J. Wang, S. Balakirsky, and C. Scrapper. Bridging the gap between simulation and reality in urban search and rescue. In Robocup 2006: Robot Soccer World Cup X. Springer, LNAI, 2006.

3. M.W.M.G. Dissanayake, P. Newman, S. Clark, H.F. Durrant-Whyte, and M. Csorba. A solution to the simultaneous localization and map building (slam) problem. IEEE Transactions on Robotics and Automation, 17(3):229-241, 2001.

4. J. Djugash, S. Singh, and P. Corke. Further results with localization and mapping using range from radio. In Proc. of the Fifth Int. Conf. on Field and Service Robotics, Pt. Douglas, Australia, 2005. 
5. M. A. Fischler and R. C. Bolles. Random sample consensus: A paradigm for model fitting with applications to image analysis and automated cartography. Comm. of the ACM, 24:381-395, 1981.

6. M.S. Grewal, L. R. Weill, and A. P. Andrews. Global Positioning Systems, Inertial Navigation, and Integration. John Wiley \& Sons, 2001.

7. D. Hähnel, W. Burgard, D. Fox, K. Fishkin, and M. Philipose. Mapping and localization with rfid technology. In Proc. of the IEEE International Conference on Robotics and Automation (ICRA), 2004.

8. Texas Instruments. Datasheet, "cc2420 2.4 ghz ieee 802.15.4 / zigbee-ready rf transceiver, rev swrs041, 2006. http://www. chipcon.com.

9. G. Kantor, S. Singh, R. Peterson, D. Rus, A. Das, V. Kumar, G. Pereira, and J. Spletzer. Distributed search and rescue with robot and sensor team. In Proc. of the Int. Conf. on Field and Service Robotics (FSR), pages 327-332. Sage Publications, July 2003.

10. A. Kehagias, J. Djugash, and S. Singh. Range-only slam with interpolated range data. Technical Report CMU-RI-TR-06-26, Robotics Institute, Carnegie Mellon University, 2006.

11. A. Kleiner and C. Dornhege. Real-time localization and elevation mapping within urban search and rescue scenarios. Journal of Field Robotics, 24(8-9):723-745, 2007.

12. A. Kleiner, B. Steder, C. Dornhege, D. Hoefler, D. Meyer-Delius, J. Prediger, J. Stueckler, K. Glogowski, M. Thurner, M. Luber, M. Schnell, R. Kuemmerle, T. Burk, T. Braeuer, and B. Nebel. Robocuprescue - robot league team rescuerobots freiburg (germany). In RoboCup 2005 (CDROM Proceedings), Team Description Paper, Rescue Robot League, Osaka, Japan, 2005.

13. A. Kleiner and V.A. Ziparo. Robocuprescue - simulation league team rescuerobots freiburg (germany). In RoboCup 2006 (CDROM Proceedings), Team Description Paper, Rescue Simulation League, Bremen, Germany, 2006.

14. Silicon Laboratories. Datasheet, "c8051f310/1/2/3/4/5/6/7",rev 1.7 08/06, 2006. http://www.silabs.com.

15. L.E. Miller, P. F. Wilson, N. P. Bryner, Francis, J. R. Guerrieri, D. W. Stroup, and L. Klein-Berndt. Rfid-assisted indoor localization and communication for first responders. In Proc. of the Int. Symposium on Advanced Radio Technologies, 2006.

16. Michael Montemerlo. FastSLAM: A Factored Solution to the Simultaneous Localization and Mapping Problem with Unknown Data Association. PhD thesis, Robotics Institute, Carnegie Mellon University, Pittsburgh, PA, July 2003.

17. E. Olson, J. Leonard, and S. Teller. Robust range-only beacon localization. $A u$ tonomous Underwater Vehicles, 2004 IEEE/OES, pages 66-75, 2004.

18. S. Y. Seidel and T. S. Rapport. $914 \mathrm{mhz}$ path loss prediction model for indoor wireless communications in multi-floored buildings. IEEE Trans. on Antennas and Propagation, 1992.

19. R. Smith, M. Self, and P. Cheeseman. Estimating uncertain spatial relationships in robotics. Autonomous Robot Vehicles, 1:167-193, 1988.

20. T. M. Wendt and L.M. Reindl. Reduction of power consumption in wireless sensor networks through utilization of wake up strategies. In 11th WSEAS international Conference on Systems, Crete Island, Greece, 2007.

21. V.A. Ziparo, A. Kleiner, B. Nebel, and D. Nardi. Rfid-based exploration for large robot teams. In Proc. of the IEEE Int. Conf. on Robotics and Automation (ICRA), Rome, Italy, 2007. to appear. 